\title{
ELASTO-PLASTIC PLATE BENDING ANALYSIS BY $A$ BOUNDARY ELEMENT METHOD WITH INITIAL PLASTIC MOMENTS
}

\author{
A. Moshaiov \\ Department of Ocean Engineering, Massachusetts Institute of Technology, \\ Cambridge, MA 02139, U.S.A. \\ and \\ W. S. VORUS \\ Department of Naval Architecture and Marine Engineering, \\ University of Michigan, Ann Arbor, MI 48109, U.S.A.
}

(Received 17 December 1984; in revised form 20 September 1985)

\begin{abstract}
The analysis of elasto-plastic plate bending using a boundary integral equation formulation is described. The integral equations are solved using standard boundary element techniques. The plasticity, as well as the external lateral load, appears in a domain integral. The solution is obtained by an incremental loading procedure with the initial incremental plastic moments calculated by an iterative method. Several study cases are examined and good agreement is shown with published results obtained by the finite element method.
\end{abstract}

\section{NOMENCLATURE}

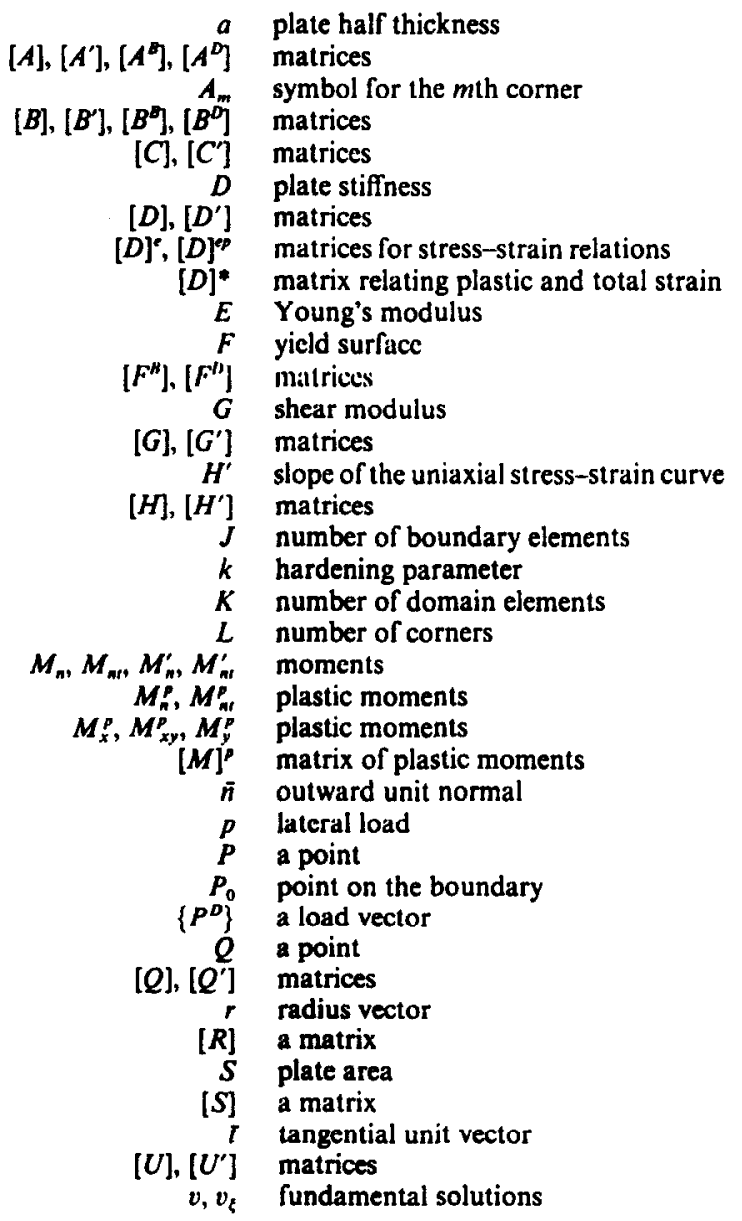




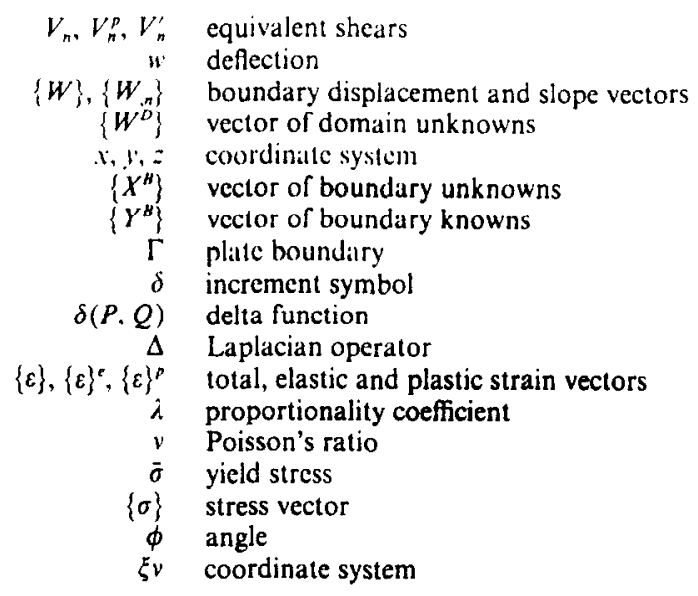

\section{INTRODUCTION}

The Green's function, or boundary integral equation method, is well known as a competitive method of solving linear elastic plate bending problems. Several authors have suggested different ways of formulating the boundary integrals for plate bending (Massonnet[1], Jawson and Maiti[2], Maiti and Chakrabarty[3], Hansen[4], Altiero and Sikarskie[5]). The formulation suggested by Bezine, and independently by Stern, has the advantage of being suitable for solving cases of general boundary conditions while the other methods are restricted to more special configurations (Bezine[6], Stern[7]). The current work extends Stern's formulation to include plasticity.

Through the use of the generalized Rayleigh-Green identity, Bezine and Stern have shown that the boundary integral equations can be obtained in terms of the relevant physical conditions existing along the boundaries. According to this direct approach, a pair of integral equations involving displacement, normal slope, bending moment and equivalent shear on the boundary are defined. Usually two of the above quantities are known for a given boundary. Generally, the suggested pair of equations is reformulated by means of boundary discretization. A discretization involving $N$ elements along the boundary creatcs a system of equations in $2 N$ unknowns.

Although the boundary element method is naturally suitable for linear problems, it is also useful for solving elasto-plastic problems of two and three dimensions by means of initial strain or stress (Banerjee and Butterfield[8]). Usually when using these methods an incremental approach is applied in which the load is divided into increments. An iterative method is then used to evaluate the plastic strain existing at each increment. This basic approach of successive elastic approximations is commonly used in solving elasto-plastic problems by the finite element method, and has been used with the boundary element method. A positive feature is that the matrix involved in the solution does not change during the incrementation and can therefore be inverted in advance and retained.

A boundary element solution for the nonlinear plate bending problem was recently suggested and demonstrated by Morjaria and Mukherjee[9]. The formulation given there has the major drawback that only restricted boundary conditions can be used. The current paper extends the general elastic plate bending formulation of Stern to the elasto-plastic case (Stern[7]). It is shown that this extension retains the advantage of dealing with the physical quantities of displacement, normal slope, normal moment and equivalent shear along the boundary.

Morjaria and Mukherjee used numerical second derivatives of the nonlinear strains in their formulation. In this paper, the problem is formulated such that no derivatives of the plastic strains are needed.

Three study cases are described and a comparison with published results obtained by the finite element method are presented (Popov et al.[10, 11], Owen and Figueiras[12]). 


\section{GOVERNING EQUATION}

Following Kirchoff's assumptions based on the small deflections theory of thin plates, the biharmonic equation for the elastic plate bending can be reformulated in incremental form to include the plastic strain increments. It is assumed that any in-plane effects are neglected and that the midplane of the plate is a plane of symmetry even in the presence of plastic strains.

The increment of the total strain can be written as a superposition of increments of the elastic strain and the plastic strain as follows:

$$
\delta\{\varepsilon\}=\delta\{\varepsilon\}^{e}+\delta\{\varepsilon\}^{p}
$$

For the elastic component under plane stress :

$$
\begin{aligned}
& \delta \sigma_{x x}=\frac{E}{1-v^{2}}\left(\delta \varepsilon_{x x}^{e}+v \delta \varepsilon_{y y}^{e}\right), \\
& \delta \sigma_{y y}=\frac{E}{1-v^{2}}\left(\delta \varepsilon_{y y}^{e}+v \delta \varepsilon_{x x}^{e}\right), \\
& \delta \sigma_{x y}=2 G \delta \varepsilon_{x y}^{e} .
\end{aligned}
$$

According to the kinematic assumptions the increments of the total strain are given in terms of the partial derivatives of the deflection increment as follows:

$$
\begin{aligned}
& \delta \varepsilon_{x x}=-z \delta w_{, x x}, \\
& \delta \varepsilon_{y y}=-z \delta w_{, y y}, \\
& \delta \varepsilon_{x y}=-z \delta w_{x y} .
\end{aligned}
$$

Following the derivation procedure of the elastic plate bending equation, the incremental form of the equilibrium equation gives

$$
\frac{\partial^{2} \delta M_{x}}{\partial x^{2}}+2 \frac{\partial^{2} \delta M_{x y}}{\partial x \partial y}+\frac{\partial^{2} \delta M_{y}}{\partial y^{2}}+\delta p=0
$$

where the increments of the moments are defined as

$$
\begin{aligned}
\delta M_{x} & =\int_{-a}^{a} \delta \sigma_{x x} z \mathrm{~d} z, \\
\delta M_{y} & =\int_{-a}^{a} \delta \sigma_{y y} z \mathrm{~d} z \\
\delta M_{x y} & =\int_{-a}^{a} \delta \sigma_{x y} z \mathrm{~d} z .
\end{aligned}
$$

Using eqns (1) and (2) the stresses can be expressed as follows:

$$
\begin{aligned}
\delta \sigma_{x x} & =\frac{E}{1-v^{2}}\left(\delta \varepsilon_{x x}+v \delta \varepsilon_{y y}\right)-\frac{E}{1-v^{2}}\left(\delta \varepsilon_{x x}^{p}+v \delta \varepsilon_{y y}^{p}\right), \\
\delta \sigma_{y y} & =\frac{E}{1-v^{2}}\left(\delta \varepsilon_{y y}+\nu \delta \varepsilon_{x x}\right)-\frac{E}{1-v^{2}}\left(\delta \varepsilon_{y y}^{p}+\nu \delta \varepsilon_{x x}^{p}\right), \\
\delta \sigma_{x y} & =2 G \delta \varepsilon_{x y}-2 G \delta \varepsilon_{x y}^{p} .
\end{aligned}
$$


Substituting eqn (6) into eqn (5), the increments of the plastic moments become defined as

$$
\begin{aligned}
& \delta M_{x}^{p}=\int_{-a}^{a} \frac{E}{1-v^{2}}\left(\delta \varepsilon_{x x}^{p}+v \delta \varepsilon_{y, y}^{p}\right) z \mathrm{~d} z, \\
& \delta M_{y}^{p}=\int_{a}^{a} \frac{E}{1-v^{2}}\left(\delta \varepsilon_{y y}^{p}+v \delta \varepsilon_{x, x}^{p}\right) z \mathrm{~d} z, \\
& \delta M_{x y}^{p}=\int_{-a}^{a} 2 G \delta \varepsilon_{x y}^{p} z \mathrm{~d} z .
\end{aligned}
$$

Substituting the plastic and elastic moment increments into the equilibrium eqn (4) and using eqns (3), (5), (6) and (7), the governing equation for the elasto-plastic plate bending in an incremental form is obtained:

$$
D \Delta \Delta \delta w=\delta p-\nabla \nabla \delta[M]^{p},
$$

where $\Delta$ is the Laplacian operator and $\nabla$ is defined as

$$
\nabla=\left[\frac{\partial}{\partial x}, \frac{\partial}{\partial y}\right]
$$

also

$$
\delta[M]^{p}=\left[\begin{array}{ll}
\delta M_{x}^{p} & \delta M_{x y}^{p} \\
\delta M_{x y}^{p} & \delta M_{y}^{p}
\end{array}\right]
$$

The plate bending equation as obtained in eqn (8) includes lateral loading and plastic effects. The plastic moment tensor, which must be unknown at any increment, simply appears in the equation as an additional effective lateral load.

Next, following a short description of the constitutive relations, the governing equation, (8), is transformed into a boundary integral form, with special treatment required for the plasticity terms.

\section{CONSTITUTIVE RELATIONS}

The elasto-plastic analysis requires appropriate constitutive relations. The Prandt1Reuss stress strain relations based on Von Mises' yield condition is used. The formulation given by Zienkiewicz et al.[13] is adopted.

A general yield surface $F$, is given in terms of the stress space and a hardening parameter $k$,

$$
F(\{\sigma\}, k)=0
$$

Using the normality principle, require:

$$
\delta\{\varepsilon\}^{p}=\lambda \frac{\partial F}{\partial\{\sigma\}}
$$

Rewriting eqn (2) in matrix form using $[D]^{e}$ as the elasticity matrix and combining the increments of the elastic and the plastic strain results in the increment of the total strain as follows:

$$
\delta\{\varepsilon\}=[D]^{e^{-1}} \delta\{\sigma\}+\lambda \frac{\partial F}{\partial\{\sigma\}}
$$


Plasticity occurs on the yield surface $F$ of the stress space. This requires:

$$
0=\left\{\frac{\partial F}{\partial\{\sigma\}}\right\}^{T} \delta\{\sigma\}+\frac{\partial F}{\partial k} \mathrm{~d} k
$$

Using eqns (13) and (14), the following stress-strain relationship is obtained,

$$
\delta\{\sigma\}=[D]^{e p} \delta\{\varepsilon\}
$$

where

$$
[D]^{e p}=[D]^{e}-[D]^{e}\left\{\frac{\partial F}{\partial\{\sigma\}}\right\}\left\{\frac{\partial F}{\partial\{\sigma\}}\right\}^{T}[D]^{c}\left(H^{\prime}+\left\{\frac{\partial F}{\partial\{\sigma\}}\right\}^{T}[D]^{e}\left\{\frac{\partial F}{\partial\{\sigma\}}\right\}\right)^{-1}
$$

It can be shown that $H^{\prime}$, in eqn (16), is the slope of the uniaxial stress $\bar{\sigma}=\bar{\sigma}(k)$ versus the uniaxial plastic strain as obtained in a uniaxial yield test. The Von Mises' yield surface for the plane stress case is given as

$$
F=\left[\sigma_{x x}^{2}+\sigma_{y y}^{2}-\sigma_{x x} \sigma_{y y}+3 \sigma_{x y}^{2}\right]^{1 / 2}-\bar{\sigma}
$$

Equations (1), (2) and (15) are used to arrive at the equation

$$
\delta\{\varepsilon\}^{p}=[D]^{*} \delta\{\varepsilon\}
$$

where

$$
\begin{gathered}
{[D]^{*}=\left[\Pi-[D]^{e^{-1}}[D]^{e p}\right.} \\
{[D]^{*}=\left\{\frac{\partial F}{\partial\{\sigma\}}\right\}\left\{\frac{\partial F}{\partial\{\sigma\}}\right\}^{T}[D]^{e}\left(H^{\prime}+\left\{\frac{\partial F}{\partial\{\sigma\}}\right\}^{T}[D]^{e}\left\{\frac{\partial F}{\partial\{\sigma\}}\right\}\right)^{-1}}
\end{gathered}
$$

Equation (18) is needed for the iterative procedure. It relates the increments of the plastic strain to the increments of the total strain. The plastic strains are required in eqn (7), and subsequently in the integral equation solution for $w(x, y)$ as presented in the following sections.

\section{THE BOUNDARY INTEGRALS}

The generalized Rayleigh-Green identity has been used in the past to obtain boundary integral equations for the biharmonic equation governing elastic plate bending (Bezine[6], Stern[7]). The identity can be written in incremental form as follows:

$$
\begin{aligned}
\int_{S} \int(v \Delta \Delta \delta w-\delta w \Delta \Delta v) \mathrm{d} S= & -D^{-1} \int_{\Gamma}\left\{v \delta V_{n}^{\prime}(\delta w)-\delta w V_{n}(v)\right\} \mathrm{d} s \\
& -D^{-1} \int_{\Gamma}\left\{\frac{\partial}{\partial n}(\delta w) M_{n}(v)-\frac{\partial v}{\partial n} \delta M_{n}^{\prime}(\delta w)\right\} \mathrm{d} s \\
& +D^{-1} \sum_{m=1}^{L}\left\{\left[\delta w M_{n t}(v)\right]_{\Lambda_{m}}-\left[v \delta M_{n t}^{\prime}(\delta w)\right]_{A_{m}}\right\}
\end{aligned}
$$

The above relation holds for all functions $v$ and $\delta w$ which are four times continuously differentiable. Let $S$ be the domain occupied by the plate and $\Gamma$ be its boundary. Also let $S$ be the arc length along the boundary, $\bar{n}$ the outward unit normal vector and $t$ the unit 
vector in the tangential direction of the boundary (see Fig. 1 for notation). On the boundary $\Gamma$ define $M_{n}, M_{n t}$ and $V_{n}$ as the actual distributions of bending moment, twisting moment and equivalent transverse shear force, respectively. The corresponding components $M_{n}^{\prime}$, $M_{n i}^{\prime}$ and $V_{n}^{\prime}$ are defined as functions of the deflection field $\delta w$ as obtained in an elastic case. In other words, the above functions can be obtained from eqns (3), (2) and (5) when the total strain is taken equal to the elastic strain. Also $M_{n}(v), M_{n t}(v)$ and $V_{n}(v)$ are defined as in the elastic case corresponding to a deflection field $v$. The last summation term in the above equation is a result of possible discontinuities in $L$ corners. The jump at a corner $A_{m}$ is defined by

$$
[\cdot]_{A_{m}}=(\cdot)_{A_{m}^{+}}-(\cdot)_{A_{m}^{-}} \cdot
$$

Consider two points $P\left(x_{P}, y_{P}\right)$ and $Q\left(x_{Q}, y_{Q}\right)$ of the region $S$ and let

$$
v(P, Q)=\frac{1}{8 \pi} r^{2} \log r
$$

where

$$
r=\left\{\left(x_{Q}-x_{P}\right)^{2}+\left(y_{Q}-y_{P}\right)^{2}\right\}^{-1 / 2}
$$

The Green's function $v$ is a fundamental solution such that

$$
\Delta \Delta v(P, Q)=\delta(P, Q)
$$

Substitute the governing eqn (8) and the above eqn (24), into eqn (20), and get for any internal point $P$,

$$
\begin{aligned}
\delta w(P)= & D^{-1} \int_{\Gamma}\left\{v \delta V_{n}^{\prime}(\delta w)-\frac{\partial v}{\partial n} \delta M_{n}^{\prime}(\delta w)+\frac{\partial}{\partial n}(\delta w) M_{n}(v)-\delta w V_{n}(v)\right\} \mathrm{d} s_{Q} \\
& +D^{-1} \int_{S} \int v\left\{\delta p-\nabla \nabla \delta[M]^{p}\right\} \mathrm{d} S_{Q}-D^{-1} \sum_{m=1}^{L}\left\{\left[\delta w M_{n t}(v)\right]_{A_{m}}-\left[v \delta M_{n t}^{\prime}(\delta w)\right]_{A_{m}}\right\}
\end{aligned}
$$

Here, $\mathrm{d} S_{Q}$ and $\mathrm{d} s_{Q}$ denote integration over $S$ and $\Gamma$ with respect to the coordinates of point $Q$. Equation (25) is of an inconvenient form since it requires derivatives of the plastic moments and it does not include the actual physical components along the boundary. A better form can be achieved by the following integration by parts :

$$
\begin{aligned}
\int_{S} \int v \nabla \nabla \delta[M]^{p} \mathrm{~d} S=\int_{S} \int\left\{\frac{\partial^{2} v}{\partial x^{2}} \delta M_{x}^{p}+2 \frac{\partial^{2} v}{\partial x \partial y} \delta M_{x y}^{p}+\frac{\partial^{2} v}{\partial y^{2}} \delta M_{y}^{p}\right\} \mathrm{d} S \\
-\int_{\Gamma}\left(\delta M_{n}^{p} \frac{\partial v}{\partial n}-v \delta V_{n}^{p}\right) \mathrm{d} s+\sum_{m=1}^{L}\left[v \delta M_{n]}^{p}\right] .
\end{aligned}
$$

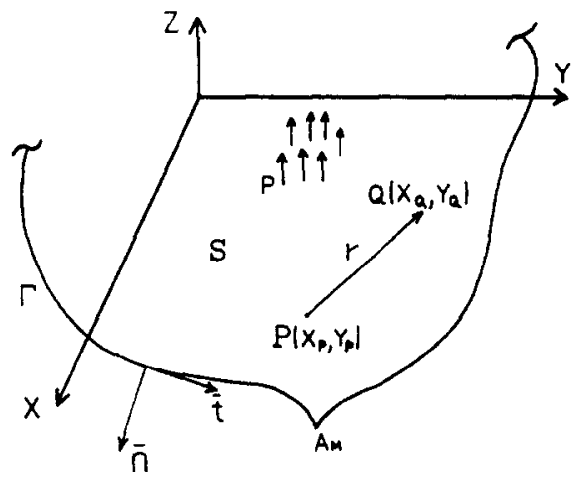

Fig. 1. Notation for the boundary integrals formulation. 
Substituting eqn (26) into eqn (25) results in

$$
\begin{aligned}
\delta w(P)= & D^{-1} \int_{\Gamma}\left\{v\left(\delta V_{n}^{\prime}(\delta w)-\delta V_{n}^{p}\right)-\delta w V_{n}(v)\right\} \mathrm{d} s_{Q} \\
& +D^{-1} \int_{\Gamma}\left\{\frac{\partial}{\partial n}(\delta w) M_{n}(v)-\frac{\partial v}{\partial n}\left(\delta M_{n}^{\prime}(\delta w)-\delta M_{n}^{p}\right)\right\} \mathrm{d} s_{Q} \\
& -D^{-1} \int_{S} \int\left\{\frac{\partial^{2} v}{\partial x^{2}} \delta M_{x}^{p}+2 \frac{\partial^{2} v}{\partial x \partial y} \delta M_{x y}^{p}+\frac{\partial^{2} v}{\partial y^{2}} \delta M_{y}^{p}-\delta p v\right\} \mathrm{d} S_{Q} \\
& +D^{-1} \sum_{m=1}^{L}\left\{\left[\delta w M_{n r}(v)\right]_{A_{m}}-\left[v\left(\delta M_{n t}^{\prime}(\delta w)-\delta M_{n t}^{p}\right)\right]_{A_{m}}\right\} .
\end{aligned}
$$

Using eqns (5), (6) and (7) the increment of the bending moment $\delta M_{n}$ normal to the boundary is

$$
\delta M_{n}=\delta M_{n}^{\prime}-\delta M_{n}^{\nu}
$$

Similarly it can be shown that the increment of the effective shear force $\delta V_{n}$ on the boundary is

$$
\delta V_{n}=\delta V_{n}^{\prime}-\delta V_{n}^{p}
$$

and the increment of the twisting moment $\delta M_{n}$ is

$$
\delta M_{n t}=\delta M_{n t}^{\prime}-\delta M_{n t}^{p} .
$$

Therefore eqn (27) can be rewritten as

$$
\begin{aligned}
\delta w(P)= & D^{-1} \int_{\Gamma}\left\{v \delta V_{n}(\delta w)-\delta w V_{n}(v)\right\} \mathrm{d} s_{Q} \\
& +D^{-1} \int_{\Gamma}\left\{\frac{\partial}{\partial n}(\delta w) M_{n}(v)-\frac{\partial v}{\partial n} \delta M_{n}\left(\delta w^{\prime}\right)\right\} \mathrm{d} s_{Q} \\
& -D^{-1} \int_{S} \int\left\{\frac{\partial^{2} v}{\partial x^{2}} \delta M_{x}^{p}+2 \frac{\partial^{2} v}{\partial x \partial y} \delta M_{x y}^{p}+\frac{\partial^{2} v}{\partial y^{2}} \delta M_{y}^{p}-\delta p v\right\} \mathrm{d} S_{Q} \\
& +D^{-1} \sum_{m=1}^{L}\left\{\left[\delta w M_{m}(v)\right]_{A_{m}}-\left[v \delta M_{n !}(\delta w)\right]_{A_{m}}\right\} .
\end{aligned}
$$

Equation (31) gives the value of $\delta w$ at any interior point $P$ of $S$ in terms of the physical quantities $\delta w,(\partial / \partial n)(\delta w), \delta M_{n}$ and $\delta V_{n}$ on $\Gamma$.

Now let point $P$ tend to $P_{0}$ on $\Gamma$. Taking the limiting values of the integrals, the following equation for point $P_{0}$ on $\Gamma$ is obtained:

$$
\begin{aligned}
0.5 \delta w\left(P_{0}\right)= & D^{-1} \int_{\Gamma}\left\{v \delta V_{n}(\delta w)-\delta w V_{n}(v)\right\} \mathrm{d} s_{Q} \\
& +D^{-1} \int_{\Gamma}\left\{\frac{\partial}{\partial n}(\delta w) M_{n}(v)-\frac{\partial v}{\partial n} \delta M_{n}(\delta w)\right\} \mathrm{d} s_{Q} \\
& -D^{-1} \int_{S} \int\left\{\frac{\partial^{2} v}{\partial x^{2}} \delta M_{x}^{p}+2 \frac{\partial^{2} v}{\partial x \partial y} \delta M_{x y}^{p}+\frac{\partial^{2} v}{\partial y^{2}} \delta M_{y}^{p}-\delta p v\right\} \mathrm{d} S_{Q} \\
& +D^{-1} \sum_{m=1}^{L}\left\{\left[\delta w M_{n t}(v)\right]_{A_{m}}-\left[v \delta M_{n t}(\delta w)\right]_{A_{m}}\right\},
\end{aligned}
$$

where $v=v\left(P_{0}, Q\right)$. 
A second independent equation is needed since two conditions will always be unknown on the boundary. According to Stern a second equation, for the normal derivative of $u^{\prime}$ at point $P_{0}$ along the boundary, can be derived as follows (Stern[7]). A local $\xi \eta$-coordinate system is introduced at a point $P$, as illustrated in Fig. 2. The following fundamental solution is used:

$$
V_{\xi}=\frac{1}{2 \pi} r \ln r \cos \phi,
$$

where $\phi$ is the angle of rotation of $r$ with respect to the $\xi$ direction. It is noted that the ahove fundamental solution gives the following kernel due to the equivalent shear:

$$
V\left(v_{\xi}\right)=-D \frac{3-v}{2 \pi} \frac{\cos \phi}{r^{2}} \text {. }
$$

Due to the $l / r^{2}$ behavior of this kernel, as $Q$ approaches $P_{0}$ the boundary integral does not converge in general. Stern suggested substituting $w-w(P)$ into the generalized RayleighGreen identity to achieve convergence (Stern[7]). The second boundary integral equation for a regular point $P_{0}$ along the boundary as given by Stern is herewith modified to incremental form with the inclusion of the plastic effects:

$$
\begin{aligned}
-\frac{\partial}{\partial n}\left(\delta w\left(P_{0}\right)\right)= & D^{-1} \int_{\Gamma}\left\{v_{\xi} \delta V_{n}^{\prime}(\delta w)-\frac{\partial v_{\xi}}{\partial n} \delta M_{n}^{\prime}(\delta w)\right\} \mathrm{d} s_{Q} \\
& +D^{-1} \int_{\Gamma}\left\{\frac{\partial}{\partial n}(\delta w) M_{n}\left(v_{\xi}\right)-\left(\delta w-\delta w\left(P_{0}\right)\right) V_{n}\left(v_{\xi}\right)\right\} \mathrm{d} s_{Q} \\
& +D^{-1} \int_{S} \int v_{\xi}\left\{\delta p-\nabla \nabla \delta[M]^{p}\right\} \mathrm{d} S_{Q} \\
& +D^{-1} \sum_{m=1}^{L}\left\{\left[\left(\delta w-\delta w\left(P_{0}\right)\right) M_{n t}\left(v_{\xi}\right)\right]_{A_{m}}-\left[v_{\xi} \delta M_{n t}^{\prime}(\delta w)\right]_{A_{m}}\right\}
\end{aligned}
$$

where $\xi$ is taken in the normal direction to the boundary at $P_{0}$. Replacing $v$ with $v_{\xi}$ in eqn (26) and substituting into eqn (35), the following equation is obtained:

$$
\begin{aligned}
-\frac{\partial}{\partial n}\left(\delta w\left(P_{0}\right)\right)= & D^{-1} \int_{\Gamma}\left\{v_{\xi} \delta V_{n}(\delta w)-\left(\delta w-\delta w\left(P_{0}\right)\right) V_{n}\left(v_{\xi}\right)\right\} \mathrm{d} s_{Q} \\
& +D^{-1} \int_{\Gamma}\left\{\frac{\partial}{\partial n}(\delta w) M_{n}\left(v_{\xi}\right)-\frac{\partial v_{\xi}}{\partial n} \delta M_{n}(\delta w)\right\} \mathrm{d} s_{Q} \\
& -D^{-1} \int_{S} \int\left\{\frac{\partial^{2} v_{\xi}}{\partial x^{2}} \delta M_{x}^{p}+2 \frac{\partial^{2} v_{\xi}}{\partial x \partial y} \delta M_{x y}^{p}+\frac{\partial^{2} v_{\xi}}{\partial y^{2}} \delta M_{y}^{p}-\delta p v_{\xi}\right\} \mathrm{d} S_{Q} \\
& +D^{-1} \sum_{m=1}^{L}\left\{\left[\left(\delta w-\delta w\left(P_{0}\right)\right) M_{n t}\left(v_{\xi}\right)\right]_{A_{m}}-\left[v_{\xi} \delta M_{n t}(\delta w)\right]_{A_{m}}\right\} .
\end{aligned}
$$

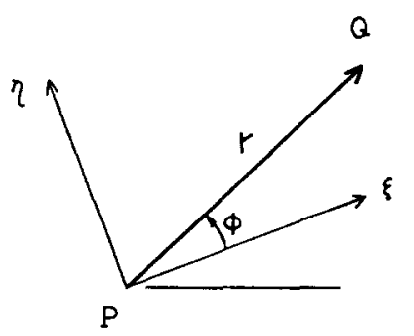

Fig. 2. Notation for $\xi v$-coordinate system 
Using eqn (32) and eqn (36), the unknowns along the boundary can be expressed in terms of the known boundary conditions, the given increment of lateral load, and the increments of the plastic moments. In general the increments of the plastic moments are unknowns but can be found by an iterative procedure. With respect to the iterative procedure it should be noted that the increments of the plastic moments are related to the increments of the plastic strains which are related to the increments of the total strains [eqn (7) and eqn (18)]. To get the increments of the total strains, equations for the second derivatives of the deflection increment are obtained. In general, the above information is needed for a point $P$ inside the domain. Equation (31) is hence differentiated twice with respect to $x_{P}$ and $y_{P}$ at the internal point $P$ giving the following equations:

$$
\begin{aligned}
\frac{\partial^{2}}{\partial x_{P}^{2}}(\delta w)= & D^{-1} \int_{\Gamma}\left\{\frac{\partial^{2} v}{\partial x_{P}^{2}} \delta V_{n}(\delta w)-\delta w \frac{\partial^{2}}{\partial x_{P}^{2}}\left(V_{n}(v)\right)\right\} \mathrm{d} s_{Q} \\
& +D^{\prime} \int_{\Gamma}\left\{\frac{\partial}{\partial n}(\delta w) \frac{\partial^{2}}{\partial x_{P}^{2}}\left(M_{n}(v)\right)-\frac{\partial^{2}}{\partial x_{P}^{2}}\left(\frac{\partial v}{\partial n}\right) \delta M_{n}(\delta w)\right\} \mathrm{d} s_{Q} \\
& -D^{-1} \int_{S} \int\left\{\frac{\partial^{4} v}{\partial x_{P}^{2} \partial x_{Q}^{2}} \delta M_{x}^{p}+2 \frac{\partial^{4} v}{\partial x_{P}^{2} \partial x_{Q} \partial y_{Q}} \delta M_{x y}^{p}\right\} \mathrm{d} S_{Q} \\
& -D^{-1} \int_{S} \int\left\{\frac{\partial^{4} v}{\partial x_{P}^{2} \partial y_{Q}^{2}} \delta M_{y}^{p}-\delta p \frac{\partial^{2} v}{\partial x_{P}^{2}}\right\} \mathrm{d} S_{Q} \\
& +D^{-1} \sum_{m=1}^{L}\left\{\left[\delta w \frac{\partial^{2}}{\partial x_{P}^{2}}\left(M_{m}(v)\right)\right]_{A_{m}}-\left[\frac{\partial^{2} v}{\partial x_{P}^{2}} \delta M_{n t}(\delta w)\right]_{A_{m}}\right\},
\end{aligned}
$$

also

$$
\begin{aligned}
\frac{\partial^{2}}{\partial y_{P}^{2}}(\delta w)= & D^{-1} \int_{\Gamma}\left\{\frac{\partial^{2} v}{\partial y_{P}^{2}} \delta V_{n}(\delta w)-\delta w \frac{\partial^{2}}{\partial y_{P}^{2}}\left(V_{n}(v)\right)\right\} \mathrm{d} s_{Q} \\
& +D^{-1} \int_{\Gamma}\left\{\frac{\partial}{\partial n}(\delta w) \frac{\partial^{2}}{\partial y_{P}^{2}}\left(M_{n}(v)\right)-\frac{\partial^{2}}{\partial y_{P}^{2}}\left(\frac{\partial v}{\partial n}\right) \delta M_{n}(\delta w)\right\} \mathrm{d} s_{Q} \\
& -D^{-1} \int_{S} \int\left\{\frac{\partial^{4} v}{\partial y_{P}^{2} \partial x_{Q}^{2}} \delta M_{x}^{p}+2 \frac{\partial^{4} v}{\partial y_{P}^{2} \partial x_{Q} \partial y_{Q}} \delta M_{x y}^{p}\right\} \mathrm{d} S_{Q} \\
& -D^{-1} \int_{s} \int\left\{\frac{\partial^{4} v}{\partial y_{P}^{2} \partial y_{Q}^{2}} \delta M_{y}^{p}-\delta p \frac{\partial^{2} v}{\partial y_{P}^{2}}\right\} \mathrm{d} S_{Q} \\
& +D^{-1} \sum_{m=1}^{L}\left\{\left[\delta w \frac{\partial^{2}}{\partial y_{P}^{2}}\left(M_{n t}(v)\right)\right]_{A_{m}}-\left[\frac{\partial^{2} v}{\partial y_{P}^{2}} \delta M_{n t}(\delta w)\right]_{A_{m}}\right\}
\end{aligned}
$$

and

$$
\begin{aligned}
\frac{\partial^{2}}{\partial x_{P} \partial y_{P}}(\delta w)= & D^{-1} \int_{S}\left\{\frac{\partial^{2} v}{\partial x_{P} \partial y_{P}} \delta V_{n}(\delta w)-\delta w \frac{\partial^{2}}{\partial x_{P} \partial y_{P}}\left(V_{n}(v)\right)\right\} \mathrm{d} s_{Q} \\
& +D^{-1} \int_{\Gamma}\left\{\frac{\partial}{\partial n}(\delta w) \frac{\partial^{2}}{\partial x_{P} \partial y_{P}}\left(M_{n}(v)\right)-\frac{\partial^{2}}{\partial x_{P} \partial y_{P}}\left(\frac{\partial v}{\partial n}\right) \delta M_{n}(\delta w)\right\} \mathrm{d} s_{Q} \\
& -D^{-1} \int_{S} \int\left\{\frac{\partial^{4} v}{\partial x_{P} \partial y_{P} \partial x_{Q}^{2}} \delta M_{x}^{p}+2 \frac{\partial^{4} v^{2}}{\partial x_{P} \partial y_{P} \partial x_{Q} \partial y_{Q}} \delta M_{x y}^{p}\right\} \mathrm{d} S_{Q} \\
& -D^{-1} \int_{S} \int\left\{\frac{\partial^{4} v}{\partial x_{P} \partial y_{P} \partial y_{Q}^{2}} \delta M_{y}^{p}-\delta p \frac{\partial^{2} v}{\partial x_{P} \partial y_{P}}\right\} \mathrm{d} S_{Q} \\
& +D^{-1} \sum_{m=1}^{L}\left\{\left[\delta w \frac{\partial^{2}}{\partial x_{P} \partial y_{P}}\left(M_{n t}(v)\right)\right]_{A_{m}}-\left[\frac{\partial^{2}}{\partial x_{P} \partial y_{P}} \delta M_{m t}(\delta w)\right]_{A_{m}}\right\} .
\end{aligned}
$$


The behavior of the kernels of the domain integral as $P \rightarrow Q$ is required in the plastic moment evaluations using (37) to (39). Using Green's theorem it can be shown that

$$
\begin{gathered}
\lim _{P \rightarrow Q} \int_{S} \int \frac{\partial^{4} v}{\partial x_{P}^{2} \partial x_{Q}^{2}} \mathrm{~d} S_{Q}=\frac{3}{8}, \\
\lim _{P \rightarrow Q} \int_{S} \int \frac{\partial^{4} v}{\partial y_{P}^{2} \partial y_{Q}^{2}} \mathrm{~d} S_{Q}=\frac{3}{8}, \\
\lim _{P \rightarrow Q} \int_{S} \int \frac{\partial^{4} v}{\partial x_{P}^{2} \partial y_{Q}^{2}} \mathrm{~d} S_{Q}=\frac{1}{8}, \\
\lim _{P \rightarrow Q} \int_{S} \int \frac{\partial^{4} v}{\partial y_{P}^{2} \partial x_{Q}^{2}} \mathrm{~d} S_{Q}=\frac{1}{8}, \\
\lim _{P \rightarrow Q} \int_{S} \int_{P} \frac{\partial^{4} v}{\partial x_{P} \partial y_{P} \partial x_{Q} \partial y_{Q}} \mathrm{~d} S_{Q}=\frac{1}{8}, \\
\lim _{P \rightarrow Q} \int_{S} \int \frac{\partial^{4} v}{\partial x_{P}^{2} \partial x_{Q} \partial y_{Q}} \mathrm{~d} S_{Q}=0, \\
\lim _{P \rightarrow Q} \int_{S} \int \frac{\partial^{4} v}{\partial y_{P}^{2} \partial x_{Q} \partial y_{Q}} \mathrm{~d} S_{Q}=0, \\
\lim _{P \rightarrow Q} \int_{S} \int \frac{\partial^{4} v}{\partial x_{P} \partial y_{P} \partial y_{Q}^{2}} \mathrm{~d} S_{Q}=0, \\
\partial x_{P} \frac{\partial^{4} v}{\partial y_{P} \partial x_{Q}^{2}} \mathrm{~d} S_{Q}=0
\end{gathered}
$$

\section{NUMERICAL SOLUTION}

The boundary integrals are discretized into a linear system of algebraic equations. The boundary is approximated by $J$ straight boundary elements. Along the $j$ th element $\delta V_{n}(\delta w)$, $\delta M_{n}(\delta w),(\partial / \partial n)(\delta w)$ and $\delta w$ are assumed to be constants and are denoted as $V_{j}, M_{j}, W_{, n j}$ and $W_{j}$. It is suggested that for analyzing plates with general boundary conditions higher order interpolation functions should be used (Stern[14]). For the numerical evaluation of the domain integrals appearing in eqns (32), (36), (37), (38) and (39), the plate field is approximated by $K$ domain elements (see Fig. 3). For the $k$ th domain element the values of $\delta M_{x}^{p}, \delta M_{y}^{p}, \delta M_{x y}^{p}$ and $\delta p$ are assumed to be constants and have been denoted as $\delta M_{x k}^{p}$, $\delta M_{y k}^{p}, \delta M_{x y k}^{p}$ and $\delta p_{k}$. A detailed discussion on the incorporation of the twisting moment jumps at corners can be found in Stern[7]. For the sake of simplicity only cases with no corner effects are discussed in the following sections.

Using the above approximations eqn (32) can be written with respect to a point $P_{0}=P_{i}$ corresponding to the $i$ th element on the boundary as follows :

$$
\begin{aligned}
0.5 W_{i}=\sum_{j=1}^{J}\left(a_{i j}^{*} W_{j}\right. & \left.+b_{i j} W_{n j}+c_{i j} M_{j}+d_{i j} V_{j}\right) \\
& +\sum_{k=1}^{K}\left(g_{i k} \delta M_{x k}^{p}+h_{i k} \delta M_{y k}^{p}+q_{i k} \delta M_{x y k}^{p}+u_{i k} \delta p_{k}\right) \quad(i=1,2, \ldots, J) .
\end{aligned}
$$

Here, the coefficients are the result of integration over the corresponding elements. This 


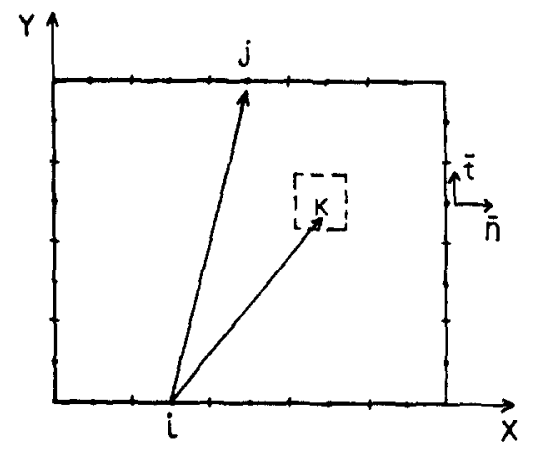

Fig. 3. Illustration of discretization method.

last set of equations, (49), can be written in matrix form as

$$
[A]\{W\}+[B]\left\{W_{n}\right\}+[C]\{M\}+[D]\{V\}=[G]\left\{\delta M_{x}^{p}\right\}+[H]\left\{\delta M_{y}^{p}\right\}+[Q]\left\{\delta M_{x y}^{p}\right\}+[U]\{\delta p\}
$$

where

$$
a_{i i}=a_{i i}^{*}-0.5 \text {. }
$$

Similarly, discretizing eqn (36) results in

$$
\begin{aligned}
-W_{, n i}=\sum_{j=1}^{J}\left(a_{i j}^{\prime *}\right. & \left.\left(W_{j}-W_{i}\right)+b_{i j}^{\prime} W_{n j}+c_{i j}^{\prime} M_{j}+d_{i j}^{\prime} V_{j}\right) \\
& +\sum_{k=1}^{K}\left(g_{i k}^{\prime} \delta M_{x k}^{p}+h_{i k}^{\prime} \delta M_{y k}^{p}+q_{i k}^{\prime} \delta M_{x y k}^{p}+u_{i k}^{\prime} \delta p_{k}\right) \quad(i=1,2, \ldots, J) .
\end{aligned}
$$

This last set of equations can be written in matrix form as

$$
\begin{aligned}
{\left[A^{\prime}\right]\{W\}+\left[B^{\prime}\right]\left\{W_{, n}\right\}+\left[C^{\prime}\right]\{M\} } & +\left[D^{\prime}\right]\{V\} \\
& =\left[G^{\prime}\right]\left\{\delta M_{x}^{p}\right\}+\left[H^{\prime}\right]\left\{\delta M_{y}^{p}\right\}+\left[Q^{\prime}\right]\left\{\delta M_{x y}^{p}\right\}+\left[U^{\prime}\right]\{\delta p\},
\end{aligned}
$$

where

$$
a_{i i}^{\prime}=-\sum_{j \neq i}^{J} a_{i j}^{\prime *}
$$

and

$$
b_{i i}^{\prime}=b_{i i}^{\prime *}+1
$$

Combine eqns (50) and (53) and rearrange them according to known and unknown boundary displacement and load conditions to obtain

$$
\left[A^{B}\right]\left\{X^{B}\right\}=\left[F^{B}\right]\left\{P^{D}\right\}+\left[B^{B}\right]\left\{Y^{B}\right\} .
$$

For example, in a clamped plate case the unknown boundary vector is $\left\{X^{B}\right\}=\{M, V\}^{T}$ and the known one is $\left\{Y^{B}\right\}=\left\{W, W_{n}\right\}^{T}$. The load vector is $\left\{P^{D}\right\}=\left\{\delta M_{x}^{p}, \delta M_{y}^{P}, \delta M_{x y}^{p}, \delta p\right\}^{T}$. Equation (56) is solved as

$$
\left\{X^{B}\right\}=\left[A^{B}\right]^{-1}\left[F^{B}\right]\left\{P^{D}\right\}+\left[A^{B}\right]^{-1}\left[B^{B}\right]\left\{Y^{B}\right\} .
$$


Using a similar approach, a vector of unknown increments of displacements and second partial derivatives inside the domain $\left\{W^{D}\right\}$ can be found using eqns (31), (37), (38) and (39) in a discretized form to be

$$
\left\{W^{D}\right\}=\left[A^{D}\right]\left\{X^{B}\right\}+\left[F^{D}\right]\left\{P^{D}\right\}+\left[B^{D}\right]\left\{Y^{B}\right\} .
$$

Substituting eqn (57) in eqn (58) results in

$$
\left\{W^{D}\right\}=[R]\left\{P^{D}\right\}+[S]\left\{Y^{B}\right\},
$$

where

$$
[R]=\left[A^{D}\right]\left[A^{B}\right]^{-1}\left[F^{B}\right]+\left[F^{D}\right]
$$

and

$$
[S]=\left[A^{D}\right]\left[A^{B}\right]^{-1}\left[B^{B}\right]+\left[B^{D}\right] .
$$

It should be noted that only $\left\{P^{D}\right\}$ in eqn (59) changes during the incremental procedure. The rest of the matrices are found in advance. Gaussian quadrature is used for evaluation of the coefficients. In the case of the domain integrals of eqns (37), (38) and (39), the limiting forms, eqns (40), (41), (42), (43) and (44), are applied in way of the singularities.

The solution procedure is :

1. Apply a load increment $\delta p$ and calculate the corresponding increments of elastic strains $\{\delta \varepsilon\}^{e}$ at the chosen integration points using eqns (58) and (3).

2. Determine increments of stress $\{\delta \sigma\}$ corresponding to $\{\delta \varepsilon\}^{e}$ by eqn (2). Define a multiplication factor $r_{y}$ which when multiplied by $\{\delta \sigma\}$ will give the elastic limit. Substitute $r_{y}\{\delta \sigma\}$ into eqn (17) and solve for the multiplication factor $r_{y}$. Repeat for all elements. Determine the multiplication factor for the onset of yielding by the smallest $r_{y}$ obtained.

3. Update $p, w,\{\varepsilon\}^{e}$ and $\{\sigma\}$ for the onset of yielding, at all domain elements and integration points, using the multiplication factor as obtained in step 3.

4. Apply a load increment $\delta p$ and assume $\delta\left[M^{p}\right]=0$ for all domain elements. If returned from step 11 , the final value of $\delta\left[M^{p}\right]$ from last increment can be chosen as a first approximation.

5. Evaluate $\{\delta \varepsilon\}$ by eqns (58) and (3) for all integration points using the assumed $\delta\left[M^{p}\right]$ and the load increment.

6. Evaluate the corresponding increments of stress $\{\delta \sigma\}$ by eqn (2).

7. Add $\{\delta \sigma\}$ to $\{\sigma\}$ and check yielding using eqn (17). When yield is checked along the plate thickness it is possible to save computational time by starting with integration points closest to the plate surfaces. Whenever yield does not occur stop checking along the thickness at that point. If the point has yielded at the start of the increment, calculate $\{\delta \varepsilon\}^{p}$ by eqn (18). If the point yields during the increment at $r\{\delta \varepsilon\}$, use $(1-r)\{\delta \varepsilon\}$ to evaluate $\{\delta \varepsilon\}^{p}$ by eqn (18) and add $r\{\delta \sigma\}$ to $\{\sigma\}$.

8. Using $\{\delta \varepsilon\}^{p}$ calculate $\delta[M]^{p}$ by eqn (7).

9. Check convergence. Convergence occurs if changes in $\delta[M]^{p}$ are sufficiently small. If convergence was not achieved replace assumed $\delta[M]^{p}$ by calculated $\delta[M]^{p}$ and return to step 5.

10. If convergence has been obtained, update $\{\sigma\},\{w\}$ and $p$. In a case of hardening, the yield stress should be updated too.

11. Return to step 4 unless $p=p_{\max }$ or a predetermined maximum number of iterations has been reached.

\section{NUMERICAL STUDIES}

Based on the above approach, a Fortran program was prepared and executed on an Apollo DN320 of the Computer Aided Engineering Network (CAEN) of the University 


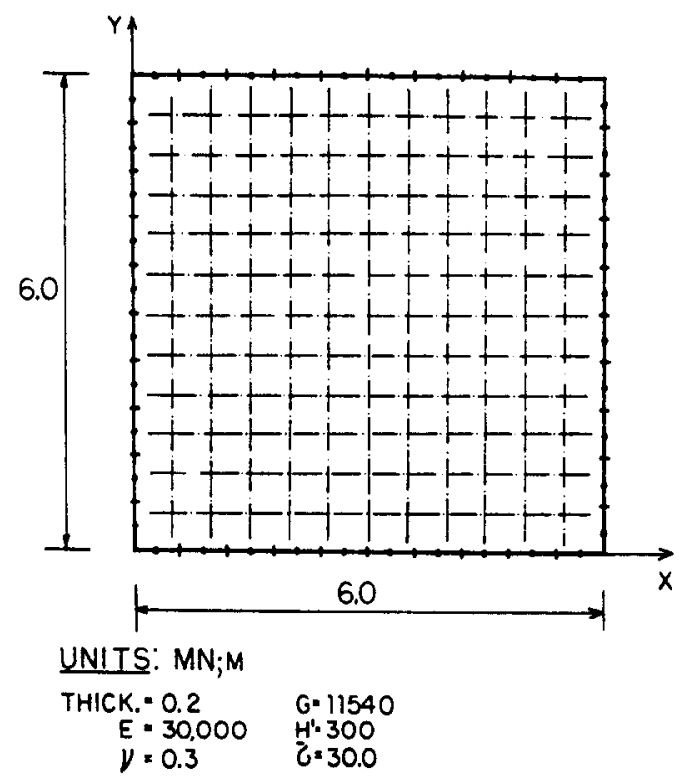

Fig. 4. Study A-Problem description.

of Michigan. Three typical elasto-plastic plate bending problems were studied to illustrate the effectiveness of the method. The first problem consists of a clamped square plate with linear strain hardening subject to uniform lateral load. The second case is an elastic perfectly plastic simply supported circular plate subject to uniform lateral load. The third case is the bending of a circular clamped plate of hardening material subject to uniform load.

Following the incremental loading procedure described in the preceding section, the deflection curves and plastic zones were found. Due to the asymptotic behavior of the deflection the number of iterations needed for each load increment increases as plasticity develops and therefore the process is generally stopped at some practical load below the limit load. The numerical studies are explained below.

Study $A$ : A clamped square plate of linear hardening material subject to uniform lateral load

A clamped square plate is subject to an increasing uniform load as described in Fig. 4. A linear strain hardening material is assumed. The boundary of the square plate is divided into $4 \times 10$ straight boundary elements of equal lengths. The domain of the plate area is divided into $12 \times 12$ square elements of equal size. Each domain element has 10 integration points along the thickness for calculating the plastic moments.

The results of the calculations are shown in Figs 5 and 6. In Fig. 5 the deflection of

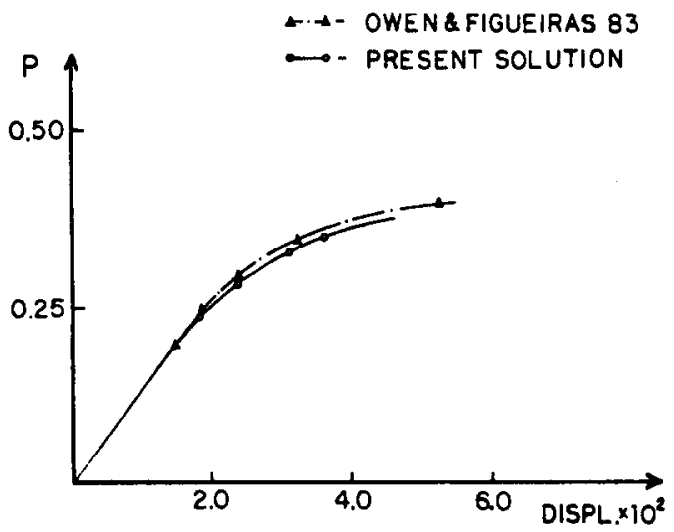

UNITS: MN;M

Fig. 5. Study A-Central deflection vs load. 


\begin{tabular}{|l|l|l|l|l|l|l|l|l|l|l|l|} 
& \multicolumn{10}{c|}{$P=0,35$} \\
\hline & & 4 & 6 & 6 & 6 & 6 & 6 & 6 & 4 & & \\
\hline 4 & 2 & 4 & 4 & & & & & 4 & 4 & 2 & \\
\hline 6 & 4 & 6 & 2 & & & & & 2 & 6 & 4 & 4 \\
\hline 6 & 4 & 2 & 4 & 4 & 2 & 2 & 4 & 4 & 2 & 4 & 6 \\
\hline 6 & & & 4 & 6 & 6 & 6 & 6 & 4 & & & 6 \\
\hline 6 & & & 2 & 6 & 6 & 6 & 6 & 2 & & & 6 \\
\hline 6 & & & 4 & 6 & 6 & 6 & 6 & 4 & & & 6 \\
\hline 6 & 4 & 2 & 4 & 4 & 2 & 2 & 4 & 4 & 2 & 4 & 6 \\
\hline 4 & 4 & 6 & 2 & & & & & 2 & 6 & 4 & 4 \\
\hline & 2 & 4 & 4 & & & & & & 4 & 2 & \\
\hline & & 4 & 6 & 6 & 6 & 6 & 6 & 6 & 4 & & \\
\hline
\end{tabular}

\# = NO. OF PLASTIC INTEGRATION PTS.

10 = TOTAL NO. OF INTEGRATION PTS. ALONG THE THICKNESS

Fig. 6. Study A-Elastic plastic boundaries.

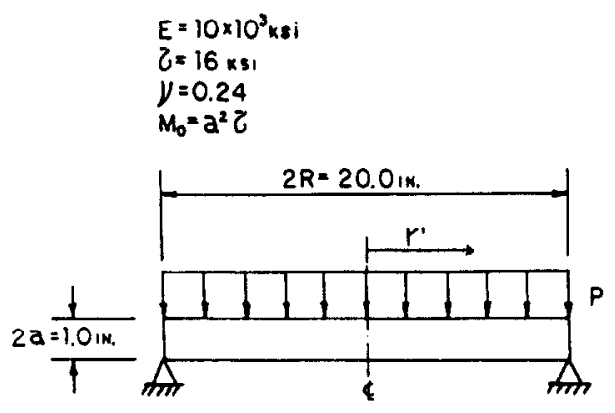

Fig. 7. Study B-Problem description.

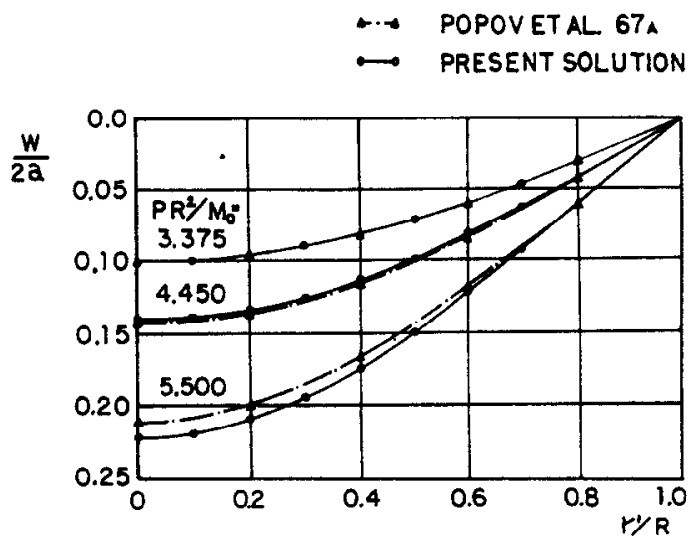

Fig. 8. Study B-Distributions of defiections. 


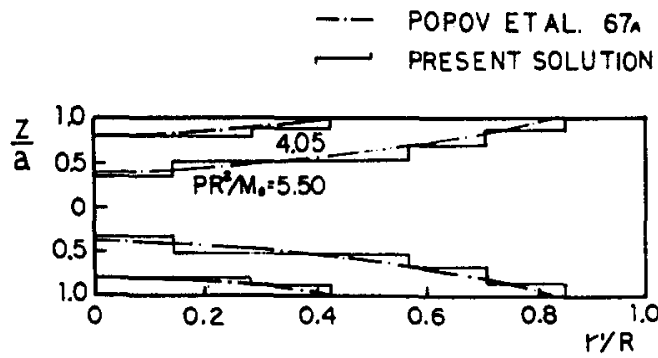

Fig. 9. Study B-Elastic plastic boundarics.

the plate center is given vs the load. The nonlinear behavior, following the linear elastic deflection, is readily observed. The good agreement with finite element results published by Owen and Figueiras[12] is obvious from Fig. 5. Figure 6 indicates the predicted spread of plasticity through the plate volume at a given load close to the limit load. The numbers appearing inside the domain elements indicate the level of plasticity through the thickness, and correspond to the number of integration points at which yielding was reached. Elements that are left blank indicate elastic zones.

Study B : Elastic perfectly plastic simply supported circular plate subject to uniform lateral load

The bending of a simply supported circular plate subject to a uniform lateral load is studied here (see Fig. 7). The plate material is taken to be elastic perfectly plastic. This example of a simply supported circular plate was studied by Popov et al.[10] using a stiffness matrix of ring elements and taking advantage of the problem symmetry. Presently, the use of symmetry is not implemented in the program developed. The plate area was approximated by 144 square and trapezoidal domain elements. It was found that the approximation associated with these element shapes had no strong effects on the results. The boundary of the plate was divided into 48 straight boundary elements of equal length. The results are shown in Figs 8 and 9. In Fig. 8 the distributions of the deflections for three load levels are given. The plasticity starts at the plate center and spreads toward the circular boundary as shown in Fig. 9. The elastic plastic boundary lines in Fig. 9. are due to Popov et al.[10] and are compared to the approximated stepped boundaries that were obtained here. Good agreement with the results obtained by Popov et al. is indicated. The small difference in the results shown in Fig. 8 is increasing with the load, and is believed to be mainly due to the flat asymptotic behavior of the deflections as plasticity spreads.

\section{Study $C$ : Clamped circular plate of hardening material subject to uniform load}

This example of a clamped circular plate of hardening material was investigated by Popov et al.[11] and is illustrated in Fig. 10. For simplicity, linear hardening is taken; this approximates the material given in the above reference (see Fig. 11). The boundary and domain elements are taken the same as in Study B described above. The results are summarized in Figs 12 and 13. In Fig. 12 the distributions of the deflections for several

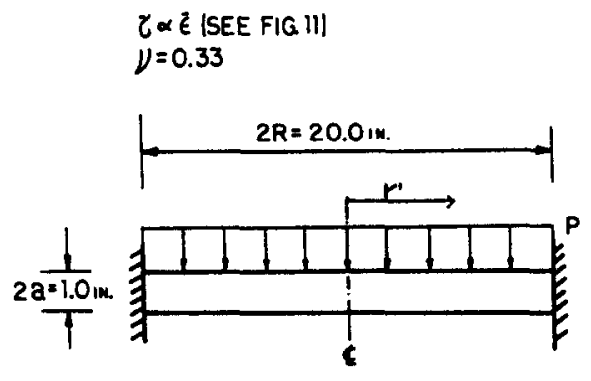

Fig. 10. Study C-Problem description. 


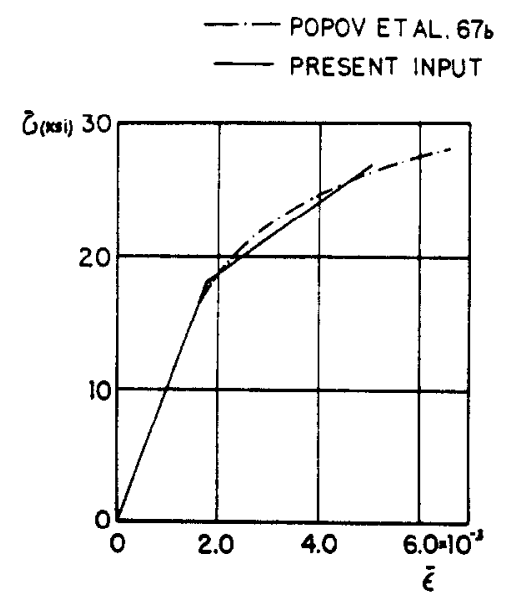

Fig. 11. Study C-Uniaxial stress-strain curve.

load levels are given. The elastic plastic boundary lines in Fig. 13 are due to Popov et al.[11] and are compared to the approximated stepped boundaries that were obtained here. The plasticity starts at the boundary and at the plate center and spreads from the plate surfaces toward the midplane as shown in Fig. 13. A good agreement is shown with the results obtained by Popov et al.[11] in spite of the linear hardening approximation used here.

\section{SUMMARY AND CONCLUSIONS}

In this work the effectiveness of boundary integrals to solve elasto-plastic plate bending problems using an incremental iterative procedure based on initial plastic moments is studied. The generalized Rayleigh-Green identity is used with the advantage of having the boundary integrals in terms of the actual physical components of deflection, slope, moment and equivalent shear along the boundary. This fact allows different admissible boundary conditions to be imposed. It is shown that the introduction of plastic moments does not change the above property.

Boundary integrals for the second partial derivatives of the deflection are formulated and used for direct calculation of the strain components needed for the evaluation of the initial plastic moments in the iterative procedure. This semianalytical method for the evaluation of the derivatives is believed to give high accuracy in comparison to the methods used for stress evaluation in finite element analysis. Some difficulties which are encountered in plate bending analysis by the finite element method are avoided by the suggested method (Zienkiewicz[15]). Other benefits of the method are the small number of unknowns and the invariance of the inverted matrix with progressive incrementation.
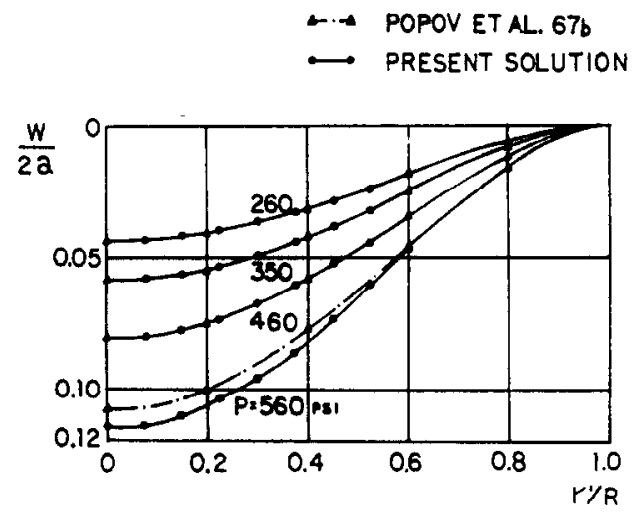

Fig. 12. Study C-Distributions of deflections. 


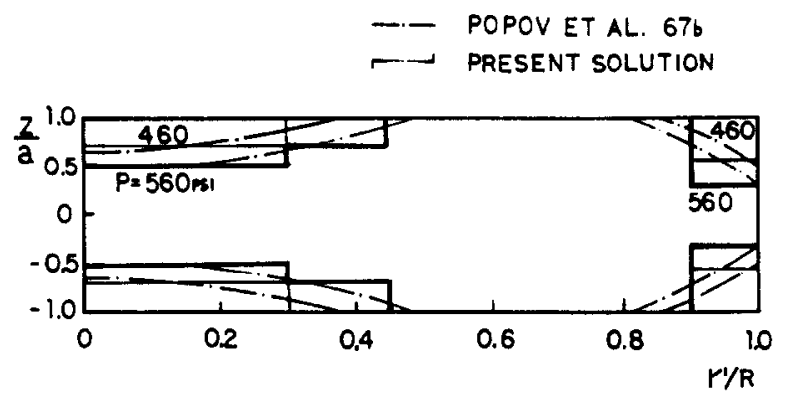

Fig. 13. Study C-Elastic plastic boundaries.

It is demonstrated through the use of several examples that the iterative method for finding the increments of the plastic moments converges. Good agreement with results obtained by the finite element method is shown.

Finally it is concluded that the method developed in this paper is capable of solving a variety of elasto-plastic plate bending problems. It can be easily used with different incremental constitutive relations and different plate shapes and boundary conditions.

Acknowledgements-This work was sponsored by the NAVSEA Cooperative Research Program in Shipbuilding Technology. The computer work was done using an Apollo DN320 on the University of Michigan Computer Aided Engineering Network (CAEN).

\section{REFERENCES}

1. C. E. Massonnet, Numerical use of integral procedures. Stress Analysis (Edited by O. C. Zienkiewicz and G. S. Holister). pp. 198-235. Wiley, London (1965).

2. M. A. Jawson and M. Maiti, An integral equation formulation of plate bending problems. J. Engng Math. 2, 83-93 (1968).

3. M. Maiti and S. K. Chakrabarty, Integral equation solutions for simply supported polygonal plates. Int. $J$. Engng Sci. 2, 793-806 (1974).

4. E. B. Hansen, Numerical solution of integrodifferential and singular integral equations for plate bending problems. J. Elasticity 6, 39-56 (1976).

5. N. J. Altiero and D. L. Sikarskic, A boundary integral method applied to plates of arbitrary plan form. Comput. Structures 9, 163-168 (1978).

6. G. Bezine, Boundary integral formulation for plate flexure with arbitrary boundary conditions. Mech. Res. Commun. 5(4), 197-206 (1978).

7. M. Stern, A general boundary integral formulation for the numerical solution of plate bending problems. Int. J. Solids Siructures 15, 769-782(1979).

8. P. K. Banerjee and R. Butterfield, Boundary Element Methods in Engineering Science. McGraw-Hill, New York (1981)

9. M. Morjaria and S. Mukherjee, Inelastic analysis of transverse deflection of plates by the boundary element method. J. Appl. Mech. 47, 291-296 (1980).

10. E. P. Popov, M. Kohjesteh-Bakht and S. Yaghmai, Analysis of elastic-plastic circular plates. J. Engng Mech. Div. Proc. Am. Soc. Civil Engrs EM6, 49-65 (1967)

11. E. P. Popov, M. Khojesteh-Bakht and S. Yaghmai, Bending of circular plates of hardening material. Int. J. Solids Structures 13, 975-988 (1967)

12. D. R. J. Owen and J. A. Figueiras, Elasto-plastic analysis of anisotropic plates and shells by the semiloof element. Int. J. Numer. Methods Engng 19, 521-539 (1983).

13. O. C. Zienkiewicz, S. Valliappan and I. P. King, Elasto-plastic solutions of engineering problems 'initial stress,' finite element approach. Int. J. Numer. Methods Engng 1, 75-100 (1969).

14. M. Stern, Boundary integral equations for bending of thin plates. In Progress in Boundary Element Methods (Edited by C. A. Brebbia). Pentech Press (1983).

15. O. C. Zienkicwicz, The Finite Element Method. McGraw-Hill, New York (1977). 